\title{
El Congreso Araucano. Ley, raza y escritura en la política mapuche
}

\author{
André MENARD, Jorge PAVEZ
}

\section{Introducción}

$\mathrm{E}$ s curioso constatar cómo la abundante literatura historiográfica y antropológica referida a la sociedad mapuche ha dejado generalmente de lado el período extendido entre la colonización definitiva del territorio mapuche por los estados chileno y argentino a fines del siglo XIX y el surgimiento de los movimientos políticos mapuches de fines del XX (década de los noventa). La intensa y densa historia política del siglo XX mapuche queda así oscurecida tras los estudios de los períodos anteriores o inmediatamente posteriores, así como por los enfoques a-historicistas de cierto culturalismo antropológico. El presente trabajo intenta abrir una perspectiva sobre este período y esta dimensión poco tratados por la mapuchografía chilena, centrándose más concretamente en las movilizaciones y debates que tensaban el movimiento mapuche en el primer tercio del siglo XX. Nos interesaremos en la institución del o los Congresos Araucanos y más especialmente en el debate surgido en 1926 en torno la promulgación de una ley de división de las comunidades mapuches.

Como veremos, el Congreso Araucano operaba a la vez como un evento o forma puntual de movilización social, y como una institución permanente de representación política mapuche. En él participaban, entre otras, las dos organizaciones políticas mapuches más importantes de esa primera mitad del siglo: la Federación Araucana liderada por Manuel 
Aburto Panguilef y la Sociedad Caupolicán Defensora de la Araucanía, primera organización política mapuche moderna fundada en 1910.

La estructuración política de este período debe ser explicada en función tanto de las circunstancias históricas vividas por la sociedad mapuche tras el proceso de conquista militar iniciado hacia 1860, así como del contexto político chileno más general. Tras la derrota definitiva en 1883, la sociedad mapuche sufre un proceso violento de reestructuración forzada. Se instala el régimen reduccional, por el cual el gobierno asigna terrenos a las familias mapuches, generando una grave pérdida económica y política. De esta forma la primera dirigencia mapuche posreduccional se criará en este contexto de crisis, caracterizado por el empobrecimiento, los abusos y sobre todo la pérdida de soberanía política. Sorprende sin embargo que a sólo una generación de la derrota, la sociedad mapuche haya logrado articular una respuesta política ante el Estado chileno para formular sus reivindicaciones. Surge así en 1910 la Sociedad Caupolicán y en 1916, la Sociedad Mapuche de Protección Mutua, que en 1921 se transformará en la Federación Araucana. Por otro lado, estas organizaciones se inscribirán en el contexto histórico chileno más general que coincide con la emergencia de las primeras formas de organización y de movilización de base popular. En este sentido resulta evidente el vínculo entre la Sociedad Mapuche de Protección Mutua y el universo mutualista chileno, como lo será, sobre todo durante la década del treinta, el vínculo entre la Federación Araucana y la Federación Obrera de Chile. Sin embargo la lógica de alianzas es más compleja. Dirigentes de la Sociedad Caupolicán, como Manuel Manquilef o Arturo Huenchullán llegarán a ser diputados por distintos partidos políticos chilenos, Liberal Democrático en el caso del primero y Democrático en el del segundo. A esto se debe agregar la a veces paradójica política de alianzas y lealtades con los gobiernos establecidos, alianzas motivadas sobre todo por el carisma de líderes como Arturo Alessandri o Carlos Ibáñez del Campo.

Ahora bien, el presente artículo se centra más específicamente en un momento preciso de la historia del Congreso Araucano. Se analizarán aquí las discusiones surgidas hacia el año 1926, en un contexto clave para el movimiento mapuche de la época. El eje de la discusión trataba fundamentalmente sobre la ley de división de comunidades propuesta ese mismo año por el diputado Manquilef, que fuera algunos años antes presidente de la Sociedad Caupolicán. Una gran parte del movimiento y de la sociedad mapuche se opondrá a esta ley, instalando así un debate que como veremos trasciende los límites de la pura cuestión económica referida a la constitución de la propiedad indígena. El y los Congresos Araucanos aparecen como un espacio privilegiado para la puesta en escena de las distintas posturas políticas que tensaron el movimiento mapuche en torno a este problema.

Cabe señalar que existe una valiosa excepción al desolador paisaje historiográfico ya descrito para este período de la historia mapuche, se trata del libro "Organizaciones, líderes y contiendas mapuches" publicado en 
1988 por Rolf Foerster y Sonia Montecino. Es sin duda la única obra dedicada a la historia política mapuche a lo largo del siglo XX (entre los años 1910 y 1970) y se basa esencialmente en el análisis de fuentes sacadas de la prensa local. Más allá del evidente valor de este libro, nos gustaría llamar la atención sobre la existencia de todo un corpus de escrituras mapuches que ha quedado fuera de este tipo de estudios. Se trata del gran número de actas, acuerdos, cartas y demás documentos producidos en el marco de estas organizaciones mapuches y de sus actividades políticas, que circulaban por redes de difusión y archivo exteriores al orden periodístico, policial o administrativo chileno. Exploraremos las líneas que vinculan este universo de escrituras mapuches con el problema de la soberanía política y sus distintas configuraciones de alianzas, conflictos y lealtades.

La escritura mapuche y el problema de la soberanía nos conducirán a otros dos puntos de importancia: primero la cuestión de la ley, vínculo privilegiado entre la escritura y la soberanía, que como veremos cruza todo el debate político de esta época, tendido entre la búsqueda de una ley que dé por fin solución al "problema mapuche", la referencia a las leyes de radicación de 1866 y 1874 y a la disyuntiva entre la igualdad de derechos y la existencia de una "legislación de la raza". Surge de lo anterior y como un segundo punto, el problema de la constitución de un sujeto colectivo, político e histórico, mapuche hipostasiado en el término "raza". De esta forma veremos cómo tras la ley y su escritura opera finalmente una pregunta sobre el cuerpo mapuche como lugar, origen, campo o vestigio en que se expresa o resiste este sujeto.

\section{La Federación Araucana y los Congresos Araucanos}

El Congreso Araucano constituyó a la vez una institución con vocación representativa y carácter resolutivo, y un conjunto de manifestaciones políticas desarrolladas entre 1921 y 1950, de ahí que el concepto oscile entre su forma singular y el plural de la serie de congresos realizados. Su origen se confunde con la formación de la Federación Araucana, una de las principales organizaciones mapuches del siglo $\mathrm{XX}$, fundada con ocasión del Primer Congreso Araucano realizado en Collimalliñ en diciembre de 1921. Detrás de esta iniciativa se encontraba Manuel Aburto Panguilef, que hasta ese momento se desempeñaba como presidente de la Sociedad Mapuche de Protección Mutua, creada cinco años antes. Según una entrevista dada al diario El Mercurio de Santiago en 1923, Aburto explica el contexto que motivó la creación de esta organización:

"Últimamente fui procurador judicial en el estudio del prestigioso abogado, señor Julio Valenzuela Campos. Así las cosas, en los últimos meses del año 1921 y con motivo de haber conocido en Santiago un proyecto de ley relacionado con los intereses materiales y estado sociológico de la raza araucana, elaborado por cierto por una persona que no entiende ni puede entender la legislación natural de esta raza, tanto por su moral 
como por sus intereses materiales, en el mes de agosto de 1921, en presencia de don Carlos A. Sadleir, de don José Andrés Huichalaf y otros en las oficinas de uno de los Ministerios, manifesté al señor Montané, el actual inspector general de Colonización e Inmigración, que no estaba de acuerdo con los demás apóstoles de esta raza que quieren la estimación completa de ella con todas sus costumbres en el país. Agregándole que sólo la voluntad soberana de la Araucanía era la llamada para pedir las leyes que le convenían y nadie más. El señor Montané celebró mi idea y me dijo que convocáramos a una reunión a todos los mapuches, cuyas conclusiones serían tomadas muy en cuenta en la elaboración del proyecto de ley por los indígenas.

En tal virtud hice que la sociedad de que era presidente, convocara al Primer Congreso Araucano en los días 24 y 25 del mes de diciembre de 1921, estableciendo entre otros puntos que debían someterse a su consideración, el que se refería a la fundación de una Federación Araucana, que con estatutos propios y ajenos a otra entidad, debía luchar en todo sentido para sublimar todas las costumbres religiosas y sociales de esta raza que nadie puede impugnar científicamente, defendiendo su moral, sus derechos que por leyes especiales les corresponden y que no a obstante ello, ha sido despojada de todas sus tierras" (El Mercurio, 20/1/1923).

En este fragmento se encuentran los elementos que en mayor o menor medida determinarán la estructura y el devenir del o de los Congresos Araucanos a lo largo de su historia. En primer lugar vemos que la Federación Araucana y su manifestación pública bajo la forma del Congreso Araucano aparecen como la respuesta a una contingencia legal referida a los "intereses materiales y estado sociológico de la raza". Será justamente en torno al campo legal, y más precisamente en torno al tema de la constitución legal de la propiedad indígena que se organizarán las discusiones políticas dentro del movimiento mapuche de la época, y más particularmente en el contexto del VI Congreso Araucano. Ahora bien, este eje temático está lejos de agotar los puntos tratados en cada una de estas reuniones así como los proyectos y construcciones ideológicas que le subyacen. Así vemos como en el fragmento recién citado aparecen al menos cuatro elementos, que si bien se engarzan con, y se subordinan al tema legislativo, trazan el horizonte ideológico que orientará toda la discusión política. En primer lugar aparece el problema de la soberanía y su destino en el marco del régimen posreduccional por el cual la sociedad mapuche es integrada al orden social chileno tras la conquista militar emprendida entre 1860 y 1885 por los Estados chileno y argentino (Pacificación de la Araucanía y Conquista del Desierto). En cierta forma podemos decir que todo el primer movimiento mapuche de principios del siglo $X X$ se puede leer como la serie de reflexiones y de respuestas prácticas ante la pérdida de esta soberanía política y territorial. En este contexto surge la cuestión legislativa como instancia de negociación y espacio de búsqueda de la soberanía perdida. Consecuentemente aparece el problema del sujeto político e histórico mapuche que supone, eleva o eclipsa la pregunta soberana. Este se 
manifiesta a través del concepto de raza y todas sus declinaciones, entre las cuales se encuentra por ejemplo el de la legislación natural de la raza reivindicada por Manuel Aburto como parámetro legitimador de las políticas legislativas que comprometan los destinos de su pueblo. Es interesante notar aquí cómo el argumento racial se inscribe en el campo científico y biologizante con que se le utilizaba en aquella época. La raza arrastra el aura de la legitimidad científicamente establecida y con ella el arsenal ideológico colonial basado en las consideraciones evolucionistas que lo justifican. Es quizás esto mismo lo que obliga a Aburto a invocar aquel otro elemento característico de su desempeño político: el argumento -que hoy llamaríamos- culturalista, por el cual se busca "sublimar todas las costumbres religiosas y sociales de esta raza" y "que nadie puede impugnar científicamente". Veremos más adelante las consecuencias que tiene esta disociación aparentemente contradictoria entre la raza, su ley natural y su moral (o sus expresiones culturales).

La Federación Araucana surge entonces como la voluntad de generar una instancia de representatividad mapuche ante el Estado chileno, instancia dotada de autonomía política, de tal forma que en su misma existencia como organización mediadora realice a su vez una forma parcial, y, como veremos, en cierta forma ritual de soberanía. Lo importante es que ésta no se agotará exclusivamente en argumentos morales y raciales, es decir, en una pura autoctonía del derecho, sino que se enraizará en el referente histórico y jurídico de las "leyes especiales", es decir, en las leyes de radicación dictadas por el Estado chileno a fines del siglo XIX ${ }^{1}$. De esta forma el campo de conformación de un espacio político mapuche se estructura en la distancia existente entre el polo de una interioridad racial y cultural y la exterioridad de un reconocimiento estatal expresado bajo la forma de un aparataje legal que se intenta afectar. Los Congresos Araucanos serán la expresión institucional de esta configuración política y en su serialidad discreta operarán una tentativa de mediación entre ambos polos de legitimación.

Los Congresos Araucanos se realizaron anualmente, a partir de 1921, por más de veinte años, en general entre el 25 de diciembre y los primeros días de enero. La elección de esta fecha no es casual. Por una parte, coincide con la época de verano, en que el tiempo es menos inclemente en la Araucanía y en que tradicionalmente se llevan a cabo los ngillatunes periódicos entre las diferentes comunidades mapuches rurales. Por otra, coincide en forma evidente con la navidad, coincidencia que no es para nada sorprendente si se toma en cuenta que Manuel Aburto Panguilef fue educado en la misión anglicana de Maquehue y que siguió durante algunos años la carrera de pastor. En este mismo sentido cabe llamara la atención sobre la presencia en aquel primer Congreso Araucano, e incluso en la redacción de los estatutos de la naciente Federación Araucana

1. Más precisamente en la ley del 4 de diciembre de 1866 sobre radicación y concesión de títulos de merced a los indígenas y la del 4 de agosto de 1874 sobre colonización extranjera por empresas particulares y prohibitiva de adquisición de terrenos de indígenas. 
del reverendo canadiense Carlos Sadleir. Este ocupará los cargos de presidente honorario de la Federación Araucana y del posterior Comité Ejecutivo de la Araucanía. Presidió -según la prensa de la época- el IV Congreso Araucano de 1924 bajo el título de Cacique General de la Araucanía (La Nación, 26/12/1924) y participó activamente en los Congresos Araucanos posteriores (incluido el VI Congreso) hasta su muerte en 1935. Si bien desconocemos las raíces políticas de su indiscutible indigenismo, debemos constatar el papel que cumplió en el desarrollo del importante componente religioso y ritual que impregnaba los congresos araucanos. Esto nos da una imagen aún más compleja de aquello que, ante la evidencia de una intensa actividad místico-ritual, algunos investigadores han clasificado como el "nativismo" o "fundamentalismo" de Manuel Aburto Panguilef, entendiéndolo como una suerte de repliegue tradicionalista en una hipotética interioridad mapuche. Sin querer iniciar aquí una discusión sobre la categoría sincrética o los avatares teológicos de la doctrina aburtiana, nos limitaremos a recalcar la lógica estratégica que guiaba al movimiento mapuche en su práctica de alianzas, pero también en la elaboración de sus enunciados y contenidos "identitarios". Vemos asimismo, confirmada por un frente wingka y cristiano, la dimensión ritual de los congresos araucanos, ritualidad que como veremos se asocia a un espacio de registro y escritura por el que se estructura un sustrato burocrático de lo mapuche.

Entre la legislación natural de la raza y el texto legal emanado del Estado chileno, el congreso araucano se despliega como una serie de eventos, una serie de congresos realizados por lo menos anualmente (en ocasiones especiales se llevaban a cabo congresos extraordinarios) en distintas localidades rurales de la Araucanía. Es como si entre ambos polos de la ley, la institucionalidad política mapuche en su búsqueda o su simulacro o su memoria de la soberanía perdida se extendiera en la concatenación de sus actualizaciones puntuales. El Congreso Araucano funciona como una suerte de reflejo inverso del Congreso Nacional. A la institución fija, inscrita en un edificio permanente, se opone esta otra, móvil, actualizada en forma intermitente, de manera itinerante y a campo abierto. Comparte con el Congreso o Parlamento nacional chileno (Aburto hablará también del Parlamento Federal de la Araucanía) el hecho de constituir un espacio de representación territorial y organizacional. Así podemos leer en las actas del VI Congreso la lista de asistentes, los que aparecen identificados con sus dos apellidos así como la localidad de donde provienen y a la que representan. Aparece así mismo su afiliación política, por llamarla de alguna manera, que cómo veremos excede en muchos casos los límites de la pura Federación Araucana. De esta forma los Congresos Araucanos intentaban articular los distintos niveles que constituían la autoridad mapuche de la época.

En 1925, durante el V Congreso Araucano, la Federación Araucana y la Sociedad Caupolicán crean en conjunto el Comité Ejecutivo de la Araucanía. Carecemos de documentos que nos precisen la función y el estatus preciso de esta entidad respecto de las otras organizaciones. Sabemos, 
eso sí, que se trata de una instancia resolutiva conjunta y que como su nombre lo indica parece asociada a una actividad más bien ejecutiva por oposición a la labor más legislativa esperable de la parte de un "congreso". Ahora bien, en los hechos esta distinción termina siendo más retórica que efectiva, ya que el Comité Ejecutivo carece de los instrumentos coercitivos y jurisdiccionales que permitirían la efectiva aplicación de sus resoluciones, y esto no sólo en lo referente al problema de la obediencia de sus bases mapuches, sino que también -y sobre todo- al reconocimiento de su autoridad por parte del Estado chileno. De esta forma el Comité Ejecutivo se nos presenta como un pliegue en la estrategia organizacional mapuche equivalente a una especie de coordinadora bicéfala de las distintas organizaciones que se dan cita en los Congresos Araucanos. Cabe sí señalar que esta pluralidad organizacional al interior de los congresos es anterior a la creación del Comité Ejecutivo, lo que los confirma en su vocación de espacio de debate ampliado más allá de los límites de la Federación Araucana.

\section{La política del registro o la soberanía por la escritura}

El otro elemento clave en estos Congresos es la política de la escritura, más precisamente la pulsión del registro. Este es quizás el principal factor de continuidad a lo largo de la serie de eventos que constituyen el Congreso Araucano. Así como su sucesión periódica asegura una primera forma de escritura a través de un ritmo y su marca calendárica del tiempo, el registro riguroso de las actas y su corolario de acuerdos, listas, telegramas, notas y circulares dirigidas a las autoridades y a la prensa, se enlaza con un flujo constante de escrituras producidas por la Federación Araucana y Comité Ejecutivo de la Araucanía a lo largo del año. Como ya ha sido señalado en trabajos anteriores, en la enorme producción escrita de la Federación Araucana y más particularmente en la de su presidente vitalicio, Manuel Aburto Panguilef, se esbozaba la constitución de un escritorio mapuche propio, análogo del territorio soberano que se buscaba recuperar, por oposición a la escritura colonizada del texto etnográfico o al terreno asignado o usurpado por la sociedad chilena bajo la forma de un Título de Merced. En otras palabras, el escritorio mapuche es a las escrituras mapuches inscritas en el escritorio etnográfico o administrativo chileno, lo que un territorio mapuche autónomo es a los terrenos inscritos en el territorio nacional chileno (Menard, 2004: 5788). El Congreso Araucano se inscribe pues en este escritorio soberano y se constituye a su vez en una suerte de enorme máquina de escritura tendida entre la escritura de las leyes y el sustrato nacional de una moralidad mapuche aparentemente exterior a la escritura y por lo tanto irreductible a todo intento de codificación. Al menos es lo que se desprende de las declaraciones hechas por Aburto en la entrevista dada al diario $L a$ Nación en 1924: "SOMOS UN GRAN PUEBLO, (...) LA LEGISLACIÓN NO ESCRITA ES MAS SABIA" (La Nación 26/12/1924, con mayúscula en el original). 
Lo curioso es que desde esta perspectiva, formalmente cercana a los culturalismos actuales y su deploración de la escritura como elemento extraño y ajeno al universo "oral" de lo mapuche, tiene el efecto paradójico de conducir a una sobreproducción de escritura. La aparente intraducibilidad del polo legal estatal y del polo moral mapuche constituye la condición de constitución de un registro y un archivo propio. Así al problema territorial considerado por el primero se vincula la cuestión de los cuerpos y su administración por el segundo (que a su vez dependerá también de la dictación de ciertas leyes). En efecto, al tiempo que exhortaba a sus miembros a no bautizar a sus hijos y reclamaba la exención para los mapuches de la obligación del carné de identidad ${ }^{2}$, la Federación Araucana llevaba un registro riguroso de sus miembros, registro que incluía fecha de nacimiento, lugar de origen, propiedades y estado civil entre otros datos. Este levantamiento de una suerte de "registro civil" autónomo se asociaba a la demanda de reconocimiento por parte del gobierno de los matrimonios realizados según "ritos indígenas" (lo que implicaba la aceptación de la poligamia) (AD 1,1: 81) así como a la demanda de respeto de los cementerios de indígenas (Ibíd.: 82).

Otra prueba de la centralidad ocupada por la escritura en el movimiento mapuche de la época aparece en el acuerdo número 25 del VIII Congreso Araucano (de Ñereco, Llaima 24-28 de diciembre de 1929), en el cual se decide recolectar fondos para adquirir una imprenta propia (ver AD 1,1: 97). Si bien este proyecto no prosperará, la voluntad de ampliar el rango mapuche de la escritura se manifestará en la sistemática producción de actas y circulares, así como en la publicación en 1935 de un órgano manuscrito, la Revista de la Federación Juvenil Araucana realizada por los miembros más jóvenes de la Federación, con la participación destacada de Cornelio Aburto Colihueque y Herminia Aburto Colihueque, hijos de Manuel Aburto ${ }^{3}$.

De esta forma vemos cómo los enunciados tradicionalistas que caracterizan cierta dimensión de los discursos emanados del Congreso reposan sobre un orden eminentemente burocrático. A la definición de la raza por la inscripción de sus cuerpos vivos y muertos en un espacio burocrático mapuche se agrega la exhaustiva trascripción de sus discursos. Las actas del VI Congreso Araucano de Ercilla constituyen en este sentido un documento excepcionalmente ilustrativo. Se trata de la descripción más detallada que se tenga de una de estas reuniones. Sorprenden tanto la profusión de detalles como la naturaleza de lo detallado. Se trata de una serie de gestos y movimientos, individuales y colectivos, normalmente acompa-

2. Ver el punto $\mathrm{N}^{\circ} 8$ de la tabla de trabajo del VI Congreso Araucano, en: Comité Ejecutivo de la Araucanía 1926.- "Actas del VI Congreso Araucano. Collico, Ercilla, $24-27$ de diciembre de 1926" - en: Anales de desclasificación, vol. 1, número 1, Santiago de Chile: Laboratorio de Desclasificación Comparada (pp. 71-95) p. 80. Gran parte de los documentos de los Congresos Araucanos que citaremos aquí corresponden al dossier publicado en estos Anales bajo el título general de Documentos de Federación Araucana y el Comité Ejecutivo de la Araucanía, comprendido entre las páginas 51 y 122. Citaremos de ahora en adelante estos documentos con el rótulo AD 1,1, seguido del número de página correspondiente.

3. Ver la reproducción de esta revista en AD 1,1: 111-122. 
ñados de un discurso más o menos transcrito por la secretaria Herminia Aburto Colihueque. Da la sensación de enfrentarse a una suerte de guión cinematográfico, o teatral, en función del cual se podría montar o reproducir el evento. Estas actas serían como la partitura de su eventual representación. Sin embargo, la proposición puede ser invertida y sostener que el Congreso es desde el comienzo una representación y la existencia de un acta no hace más que confirmar esta vocación. No existe una verdadera línea de causalidad entre el gesto y su escritura, se trata más bien de una relación de inmanencia. El acta y su congreso se reflejan mutuamente en la continuidad de un mismo plano de representación sin modelo original o parejamente originario. Da la sensación de que cada palabra y cada gesto, está funcionando en la conciencia del registro que lo trasciende.

Si bien el acta no está hecha para su representación posterior como guión teatral o cinématográfico, se construye en intertextualidad con otras representaciones políticas que jalonan la historia en forma más o menos densa. El congreso es entonces consustancial al registro, no existe fuera de la escritura que lo inscribe ni del texto mayor en que esta escritura viene a insertarse. Hay que considerar que las voces que se escuchan en el congreso hablan para ser escritas, y también que lo que se escribe se escribe para ser dicho, nuevamente representado en otros espacios de discursividad. Este aspecto queda clarísimo cuando al iniciar el cuarto día de Congreso, y después de ser elegido por unanimidad como Secretario de la mesa en reemplazo de José Inalaf Navarro (quien anuncia al presidente su partida), el profesor Ignacio Huenchullán Medel es requerido para la lectura del acta de la sesión ordinaria del quinto Congreso Araucano, acaecido en misma fecha del año anterior. Esta lectura pública del acta del Congreso anterior se realizaba con el fin de ratificar esta acta por la Asamblea en pleno. De esta manera, se lograba un primer efecto que es el de sancionar la escritura del acta, levantada y transcrita por un buró político, por parte de la Asamblea, que de esta manera afirmaba su control sobre su propia representación en un texto. El secretario iniciará la lectura a las 9:05 horas. A las 10:23 horas. Ignacio Huechullán fue reemplazado en la lectura del acta por su hermano Arturo, vicepresidente del Comité Ejecutivo, "por ser [el acta] muy extensa". El acta del VI Congreso consta entonces que a las 11:05 a.m., "se terminó con la lectura de la referida Acta [...] la que puesta en discusión en la forma de siempre, no mereció ninguna observación de ningún Delegado y se dio por aprobada.- A las once ocho minutos se suspendió por diez minutos la sesión para la firma de la Acta por los señores Presidentes i Delegados del Congreso.-" (AD 1,1: 87). Vemos cómo la lectura pública y extensiva del acta, a lo largo de dos horas cronológicas, produce dos efectos suplementarios, tan importantes como el primero: el de inscribir el V Congreso en el continuo temporal del VI Congreso, de manera a encadenar los Congresos entre ellos ${ }^{4}$, y representar así en

4. El acta de un Congreso será rubricada por la Asamblea del Congreso que sigue, de esta manera, el acta que analizamos aquí le correspondió ser leída y aprobada en el VIII Congreso de 1928, esto debido a la relegación de Manuel Aburto en 1927, lo que le impidió asistir al Congreso de 1927. 
el presente la escena del pasado, instalando por la lectura de su registro el Congreso pasado en el Congreso presente, y también instalando el Congreso futuro en el Congreso presente, por la predestinación manifiesta de su devenir como texto-para-ser-representado. En otras palabras, la lectura instaura dos movimientos: el de traer al presente los Congresos pasados y futuros, y el de hacer de la instantaneidad de este Congreso un marcador que reenvía siempre al pasado y al futuro de los Congresos Araucanos. El primer movimiento corresponde a una dimensión cronológica de la historia política, el segundo a lo que llamaremos una dimensión "ayónica" del tiempo ${ }^{5}$. Este es entonces el segundo efecto en cuestión: el de generar la conciencia de una representación política por el texto, una conciencia en torno al destino inscrito de todo acto de representación y delegación política. La política en tanto "voluntad y representación" se constituye en hecho de escritura y estas actas se vuelven entonces el guión cinematógrafico (escritura del movimiento) tanto de los afectos como de los conceptos que instituyen un espacio y una temporalidad mapuche autónoma.

De esta forma se niega la existencia de una corporalidad y de una oralidad primera y espontánea opuesta a un momento de escritura secundaria: el Congreso Araucano como entidad e institución opera desde siempre como un texto y se actualiza en su realización como evento puntual y localizado en tanto forma de escritura. Esta insistencia en la dimensión escritural o en el registro de la movilización política mapuche, no responde sólo a la necesidad de desmitificar la imagen romántica del indígena oral, tradicionalista y analfabeto, sino que también a una estrategia analítica para tratar el problema de una entidad social y política mapuche sin obviar el problema de sus límites en el contexto criptocolonial de su inscripción difusa en la sociedad chilena global. En otras palabras, el trabajo sobre textos, es decir sobre un corpus, nos permite la reflexión sobre sus alcances y sus determinaciones respecto de un "cuerpo" social y nacional mapuche concreto.

Esta condición de registro inmanente se relaciona con un paisaje histórico más extenso, que hunde sus raíces o extiende sus ramificaciones hacia los famosos parlamentos realizados entre los siglos XVIII y XIX así como hacia otras regiones del paisaje de registros e inscripciones que han trazado un espacio (un país) mapuche. Este espacio o territo-

\footnotetext{
5. Entendemos la dimensión ayónica del tiempo como aquella donde el presente es siempre escindido en pasado y futuro, trayendo a la superficie del tiempo la marca profunda del instante como escisión repetida infinitamente, en oposición a la dimensión cronológica que trae pasado y futuro a un presente donde se hunden los marcadores del tiempo. Ya no serialidad puramente cronológica de determinaciones temporales, tampoco intemporalidad o imago mundi diacrónica de un rito siempre presente y repetido, al marcar minuto a minuto la cronología del Congreso, esta termina por disolverse en el movimiento mismo de los días, los años, los siglos, que anteceden y suceden al evento. La dimensión ayónica es aquella disolución de los marcadores cronológicos en su saturación, que reenvía transversalmente a series que una cronología clásica (clasificadora) no permite cruzar. El Congreso Araucano se instala de esta manera en esa fisura ayónica del tiempo histórico. Sobre el concepto de Ayón versus Kronos, ver Deleuze (1969).
} 
rialidad textual vendrá siendo recreado y actualizado con tratados escritos, firmados con la corona de España, desde aquel decisivo parlamento de Kuyen o Quilín de 1641, y más recientemente con los no menos jurídicos, internacionales y alfabéticos debates y presentaciones en las diversas comisiones y asambleas del sistema de la Organización de las Naciones Unidas.

De hecho encontramos en la genealogía del propio Manuel Aburto la presencia de esta dimensión política de la escritura. Sabemos que su tío, Bernardo Namuncura, hijo de su abuelo Luis Ayñamco fue secretario del célebre cacique Juan Kallfükura (Calfucura) de Salinas Grandes ${ }^{6}$ y que se destacó por su intensa actividad escritural redactando cartas a las autoridades chilenas y argentinas de la época así como toda una serie de tratados de paz (Hux, 1991: 107-108). A esta función política de la escritura en el linaje de Manuel Aburto se puede agregar cierta función escritural de la política reconocible en la lógica de las alianzas y su derivación lateral de las líneas de parentesco. Así el apellido Aburto constituye la marca de un gesto de alianza establecido entre Luis Ayñamco y el comisario de naciones Francisco Aburto Ramírez a mediados del siglo XIX ${ }^{7}$. Al sellar la alianza por el traspaso de un nombre propio, éste entra en el campo de lo intercambiable, se encuentra con los nombres comunes y su capacidad figurativa, el potencial metafórico que a fin de cuentas constituye la primera forma de toda escritura ${ }^{8}$.

Como ya lo anunciamos, al registro sistemático de los gestos y actos del congreso por las actas, le corresponde la serie de gestos y actos concretos inscritos sobre el terreno de la manifestación. Se corre el awün, con caballos y hasta un vehículo, se realizan los yapepüllün, esos saltos acompañados de la exclamación "ya ya ya", se ora, pero también se dibujan sobre el mismo terreno gestos más exclusivamente políticos como la votación de la mesa de la directiva del congreso. Lla votación se realizó colocándose los partidarios de cada uno de los dos candidatos (Manuel Aburto y Luiciano Huichalaf) a uno y otro lado de la mesa en que debía sesionar el directorio. Esta modalidad de votación coreográfica al parecer no era excepcional. Germán Aburto Colihueque recordaba cómo en otro congreso, proba-

6. "De Luis Aburto Ayñamco procedía el conocido cacique civilizado Bernardo Namunkura, que murió hace pocos años, convertido como yo, a la religión evangélica. Este Namunkura fue secretario del famoso cacique Kallfükura de Salinas Grandes, en la Argentina. Se casó con una hija de éste" (Guevara \& Mañkelef 1913: 148)

7. “... nosotros somos amigos de los generales, Aburto, de la familia grande. Entonces para no guerrear más, somos descendientes de guerreros, para no guerrear más, el general se pasaron la mano y se puso Aburto, el padre de nosotros, fundador de la familia, se llamaba Aburto después... era Ayiñamco el general mapuche. Tenía sus cuarteles, sus tropas, todo eso tenía él, pero si era guerrero po. Entonces pasó a llamarse Aburto él, y el capitán de los... de amigos, el gobierno había nombrado unos capitanes de amigos (...) pa pacificar los mapuches. Capitanes de amigos se llamaban unos oficiales, graduados, distinguidos. Así que este capitán de amigos le cambió a Aburto el nombre en mapuche, le puso Aburto, se llamaba Francisco Aburto", fragmento de una entrevista realizada al difunto Germán Aburto Colihueque, hijo de Manuel Aburto en el 2002 (Menard \& Pavez 2005: 60).

8. Sobre esta relación entre escritura y metáfora ver Derrida (1967: 27); sobre la de escritura y nombre propio, Desside: 157 y ss. 
blemente el IX Congreso Araucano de Boroa realizado en 1930 o en un congreso extraordinario realizado en mayo del mismo año y en el que se discutían los pasos a seguir ante la reciente dictación de la ley de división de las comunidades, se realizó una votación "a caballo":

Porque por esa cuestión de la división de tierra pelearon mucho. Se peleó mucho aquí en la organización, porque los mapuches no estaban de acuerdo con las divisiones de tierras y los otros estaban de acuerdo. Y para aprobar la ley qué es lo que hacía. Todavía no había voto, ni cámara secreta ni nada. Don Manuel reunió toda la gente de la región, desde aquí del lado del Biobio pa'acá. Todo este conglomerado de gente. Y los hizo desfilar con su caballo, su caballería. Los que estaban de acuerdo con la división, pa'ca; los que no estaban de acuerdo, pa'l otro lado, para ver si había una mayoría. Esa fue la votación, con puros caballos no más y a jinete. No había otra manera. Por último quedó ahí no más la ley no se dividió más la propiedad. Porque si dividen la propiedad, al mapuche lo desalojan, le quitan su terreno (Menard \& Matuz, 2002).

Existe sin duda cierta mitologización arcaizante en el recuerdo de Germán Aburto. Sin embargo en esta ausencia de la cámara secreta (su lápiz y su papel podríamos agregar) y el recurso al jinete y su caballo como sujetos e instrumentos de la inscripción política, debemos ver algo más que la manifestación de un orden "prehistórico" de lo político. Por un lado se confirma la ampliación del rango del registro a la inscripción espacial de los gestos y movimientos del rito-manifestación, por otro se recurre a aquella poderosa tecnología que fue el caballo en la violenta transición del orden dromocrático (Virilio 1977) operado por la sociedad mapuche a fines del siglo XVI, y en tercer lugar, se realiza una versión a escala mapuche de aquellas grandes coreografías políticas de masas que se desarrollaron entre los años '20 y '40 en distintos contextos geográficos. De hecho, y como veremos más adelante, se puede identificar en estos congresos araucanos cierta estética futurista del primer fascio italiano o cercana al "realismo socialista", caracterizada por un trabajo de disposición y modulación de las masas en un espectáculo del poder. En este mismo sentido aparece la posición igualmente espectacular que toma la tecnología dentro de la manifestación. La tecnología ya no como simple medio o instrumento, sino que como objeto de exhibición. Es el papel jugado por la vitrola de José Inalaf Navarro en la inauguración del congreso cuando "se oyó la Cancion Nacional i la Marcial, con la Asamblea de pié i descubierta, por una Vitrola de propiedad del citado Señor Inalaf Navarro." (AD 1,1: 71). Es también el papel del automóvil facilitado por don Saturno Durán para ir a recibir a los parlamentarios a la estación ferroviaria y con el que se desarrolla una forma motorizada del ahün tradicional ${ }^{9}$. A esta espectacularización de las tecnologías se puede agregar la proliferación de telegramas enviados y recibidos a lo largo del Congreso, así como el punteo sistemático de las horas y minutos transcurridos gracias al famoso reloj de oro del presidente de la Federación Araucana.

9. "Awn: la danza y vueltas a caballo que se ejecutan alrededor del reween las rogativas o del muerto en los entierros" (Augusta 1916: 13). 
Como resultado de esta delimitación del espacio político por medio de una espectacularización de la tecnología y del acto mismo de trascripción del sujeto político constituido en Asamblea, queda el registro de la multitud concurrente al momento y espacio político. Por medio de la inscripción sistemática de los delegados, se logra la individuación de los poderes y la validación de su representatividad. Al principio del acta del Congreso se señala la presencia de "una Asamblea de más de trescientas personas". Luego, la mesa directiva abrirá el proceso de inscripción en el registro de delegados, proceso bajo la responsabilidad de Cornelio Aburto Colihueque e Ignacio Huenchullán Medel, y que logrará inscribir alrededor de 120 delegados a lo largo de los cuatro días de Congreso. Entre estos delegados se cuentan representantes de organizaciones como El Toqui de Gorbea, la Fraternidad Araucana de Gorbea, la Sociedad Caupolicán Defensora de la Araucanía, de Temuco, y la Federación Araucana, creada en Loncoche. Obviamente la organización que se impondrá por la masividad de sus representantes será la Federación Araucana y sus Consejos Federales. La lista de los delegados permite así apreciar la diseminación y arraigo territorial de la Federación Araucana. Consejos en las cuatro provincias del Ngulumapu histórico (Llanquihue, Valdivia, Cautín y Malleco). Asisten Presidentes y directivos de los Consejos de Selva Oscura (Victoria), Champulli, Chapu, Fiñ-Fiñ, Boroa, Mañio, Huincul, Raguituleufu, Malalhue-Puerto Saavedra, Chagchayen-Puerto Saavedra, Collileufu-id, Llágui-id, Peleco (todos en el departamento de Nueva Imperial), Cancha Rayada (Llanquihue), Ercilla, Huapitriu, Lolcura (del departamento de Collipulli), Maquehue, Toltén (departamento de Villarica), Riñinahue-Filuco (departamento de Río Bueno), RiachueloMaipué (departamento de Osorno) y el "Consejo Federal autónomo" de Pucura (Villarrica). A estos delegados de organizaciones políticas, se suman los caciques representantes de comunidades y sus acompañantes, o los delegados de estos mismos caciques o jefes de comunidades. Las delegaciones de las comunidades cubren un espectro territorial aún más amplio que la de los Consejos. Además, entre los nombres inscritos hay que considerar unas escasas cinco mujeres, mapuches todas, y unos no menos escasos ocho wingkas (siete chilenos y un canadiense). Sin embargo, estas minorías ocupan un rol muy activo en el congreso y posiciones de importante incidencia en el curso de los debates. Entre los wingkas, se cuentan dos senadores -Artemio Gutiérrez y Luis Enrique Concha- y un diputado -Héctor Álvarez-, el gobernador de la provincia, su secretario y un profesor -Arturo Arriagada-. Entre las mujeres, vemos viudas de caciques y dos mujeres jóvenes y militantes: Ana Huenchullán Medel y Herminia Aburto Colihueque, esta última a cargo de la redacción de las actas de este Congreso.

La precisión descriptiva de las actas, su exhaustividad y sistematicidad para registrar los cuerpos, los discursos, y las prácticas que sustentan un espacio público mapuche, permitiría realizar un análisis minucioso de la forma y modos de delimitación de un campo de acción política mapuche, de los mecanismos de validación de las representaciones, de las 
formaciones de clase y las formas de clasificación que se despliegan en un congreso araucano. Se puede leer este espacio como el lugar de confluencia y condensación de las fuerzas políticas en pugna por la hegemonía, es decir, el lugar del conflicto por la representación de la sociedad mapuche. Este conflicto por la representación deberá ser entendido en un sentido amplio: la lucha se da tanto en el ámbito de la representación de colectivos sociales (clases sociales antagónicas como son viejos / jóvenes, rurales / urbanos) y de las figuras llamadas a representar estos colectivos (caciques / profesionales), como en el ámbito de los dispositivos de la representación, es decir, la lucha por el control de los medios por los cuales los colectivos y sus agentes dejan registro público de lo representado (la Asamblea, la Raza, los Delegados).

\section{Proyectos de ley y cuerpo mapuche}

Existe una fractura importante en la sociedad política mapuche, que viene a actualizarse en torno a una coyuntura particular, la de una ley de la República de Chile sobre la constitución de la propiedad indígena. El momento es importante porque la fractura política tiene precisamente que ver con el problema de la forma y los medios de inscripción del mapuche en el marco del Estado chileno, es decir con la valoración de la legalidad estatal chilena como lugar de inscripción de lo mapuche. La crítica juvenil a la gerontocracia mapuche, la propuesta de llevar los Congresos a las ciudades, las críticas a Manuel Aburto por su veto a la presencia del diputado Manquilef, la participación de parlamentarios chilenos del Partido Demócrata, son diferentes formas de hacer visible una división que podría también llevar otros nombres (liberal / conservadores, civilizados / primitivos, campesinos / profesionales, etc.). Esta polarización, aunque no rígida, tiende a forzar la identificación de los grupos con dos proyectos para la sociedad mapuche. El debate sobre la ley nos permitirá entonces ver en que consisten tales proyectos y dimensionar el antagonismo casi irreconciliable de sus portadores.

Asumamos entonces la coyuntura legal como el eje en torno al cual chocan los dos proyectos históricos que polarizan el debate mapuche desde las leyes de radicación de 1866. Ambos proyectos están orientados a la formulación de una política de tierras que regule y fomente la constitución de la propiedad indígena. Es en torno a este problema del Estado, de su función legislativa, de su poder de coerción, y de los efectos de esta dominación sobre la estratificación social mapuche, que se enfrentan las concepciones de los dos más activos ideólogos del movimiento mapuche en este primer tercio del siglo XX. Por una parte Manuel Aburto Panguilef y por el otro Manuel Manquilef.

Cabe señalar que en un primer momento de su reflexión estos dirigentes comparten un mismo diagnóstico respecto a los efectos de las leyes de radicación de 1866 y 1884. En su manifiesto de 1915, Las Tierras de Arauco, Manquilef escribía contra la comunidad de la tierra: 
"La radicación en comunidad ha dado resultados funestos porque se igualó injustamente a todos y de aquí la lucha del más rico contra el más pobre; este exije lo que la lei le dió y aquel no puede comprender se le haya quitado lo suyo para darlo a este. Este fue un golpe premeditado al parecer contra los indios mas pudientes, a sus fortunas que quizás les habrían servido para educar sus hijos, quienes habrian defendido a su raza de tanto abuso. Mataron pues a los ricos e hicieron como los gobiernos despóticos que matan al obrero ilustrado para destruir la democracia" (Manquilef, 1915: 11).

Luego insistirá en que la comunidad de tierra servía para "tontos o santos", "como los animales" (Manquilef, 1915: 14). Once años después, declara que para quienes han sido sometidos el sistema de reducciones "la vida en comunidad les obliga a vivir peleando y los somete a la explotación inicua de los caciquillos que los representan ante los Juzgados y Protectorados respectivos" (El Diario Austral, 11/9/26). Aburto por su parte, declara en el II Congreso Araucano de 1922 que: "a sabiendas que nuestras costumbres no se prestan a una falsa asociación, dictaron una ley, tan criminal como desigual, en virtud de la cual se colocaba al ultimo mocetón en las mismas condiciones que el mas respetado de nuestros caciques" (Foerster \& Montecino 1985: 72). Ambos coinciden en la crítica del efecto igualitario de la ley por ignorar las jerarquías económicas propias de una forma nacional mapuche de estratificación. El motivo de la discordia surgirá en torno a las estrategias para salir de esta situación de subalternización general y "por abajo" de la sociedad mapuche. Aburto y Manquilef no sólo compartían un diagnóstico de la situación de clases, sino también una posición de clase: eran ambos descendientes de caciques "civilizados", ambos educados en instituciones coloniales, aunque el primero en una misión protestante y el segundo en un Liceo público laico. Seguramente esta diferencia de formación está incidiendo en la forma del conflicto. Mientras para Manquilef, la solución de los problemas mapuches pasa por su disolución como cuerpo nacional en un cuerpo mayor que es el del Estado nacional chileno, para Aburto el cuerpo colectivo mapuche tiene que producirse a sí mismo, usando para eso el Estado chileno.

Manquilef apuesta a la desaparición del "indio" en tanto producto del proceso reduccional (en 1915 señala: "Esperamos que el Gobierno cambie de rumbos y que de una vez mate a los indios y los coloque en situación de vivir como los demas ciudadanos", 1915: 37). La reducción es entendida como el principal factor de subordinación del mapuche. Su propuesta consiste en disolver la reducción para así remodelar la sociedad mapuche fusionando sus estratos con los de la sociedad chilena. Para esto es necesario un proyecto que produzca una nueva estratificación, que defina criterios de clasificación mapuche, y promueva la lógica del ascenso social. Durante la polémica de 1926, él mismo se encarga de explicar los puntos principales de su proyecto de ley:

“1. Constitución de la propiedad indígena; 2. Constitución de la familia aborigen; y 3. Clasificación de sus elementos de colectividad", 
señalando luego que el primer punto ataca el problema de la ley de 1866 donde el cacique tiene "mayor porcion a costa de los demas comuneros", el segundo es contra la poligamia ya que "con ello se pierde el derecho de herencia", y el tercer punto refiere a que "en la clasificacion se da algunas ventajas a los individuos educados para que puedan comerciar con sus suelos bajo ciertas condiciones en que la ley les considera capacitados para esto" (El Diario Austral, 8/9/26).

El clasismo de este punto será profundizado en otra entrevista donde detalla que el espíritu de este aspecto de la ley está en producir el "estimulo cultural indigena, es decir, mediante este proyecto podrán tener libre disposicion sobre sus bienes solo los indios civilizados: los semi civilizados dispondrán de sus bienes en conformidad a la ley de menores de edad y los analfabetos seguiran bajo el imperio de las leyes prohibitivas que rigen hasta hoy desde 1866" (El Diario Austral, 11/9/26). Esta estratificación debería permitir entonces fomentar el espíritu de ascenso social basado en el mérito individual de los ciudadanos, del cual es garante el Estado, ya que al haber "dividido a los indios en tres categorias para los efectos de la aplicación de la ley y la obtención de los derechos que otorga, los incorporados en la categoria de los "ignorantes" querán pasar a la de "semicivilizados", estos a su vez, a la de los "civilizados". La ley en estudio se encamina a conseguir que esos 180.000 indios se incorporen a la nacionalidad con el usufructo de todos sus derechos y garantias". (El Diario Austral, 25/12/26). El individualismo promovido por el proyecto en el marco de una estratificación de clases guiada por el manejo de la escritura vinculará al ciudadano mapuche "civilizado" al Estado bajo la forma contractualista. En el proyecto de Manquilef, la escritura se vuelve el medio para participar de este contrato en forma individual. Manquilef promueve un nacionalismo cívico chileno conservando un nacionalismo cultural mapuche productor de una objetivación racial (Pavez, 2003). La raza es lo único que queda, un resto irreductible que es un rasgo genético culturalizado por la escritura científica que lo produce y lo erige como patrimonio en el plano del humanismo universal.

El VI Congreso Araucano reaccionará a los postulados de Manquilef, proponiendo en sus resoluciones un proyecto de ley alternativo para ser presentado al Gobierno de Chile. Así podemos ver cómo a cada uno de los tres puntos en los que Manquilef resumía su propia ley, el Congreso planteará una respuesta antagónica. En primer lugar figura la rotunda negativa a la subdivisión de las comunidades y a la posibilidad de someterlas a la circulación mercantil. "LA PROPIEDAD INDIGENA DEBE SER INENAJENABLE E INEMBARGABLE" (AD 1,1: 86), dicta en este sentido y con mayúsculas el artículo 16 de dicho proyecto de ley. Más allá del cálculo económico estratégico referido a la conservación de una territorialidad mapuche por la protección de la integridad física que aseguraba la propiedad comunitaria de los terrenos, podemos identificar la idea más profunda de una inintercambiabilidad de este bien mapuche. Sería una especie de intraductibilidad de la propiedad al código general del 
mercado y sus leyes nacionales. En el segundo punto referido por Manquilef se hace mención al problema de la constitución de la familia mapuche y de las dificultades legales, concretamente sobre los derechos de herencia, que se derivan de la no regulación por el sistema legal chileno de las situaciones derivadas de la poligamia. Manquilef apostará por una regularización de esta situación a través de la aceptación de la norma civil chilena, punto retomado por el VI Congreso en sentido inverso, es decir, exigiendo el reconocimiento por el Estado chileno de los derechos derivados de la constitución de la familia indígena según la "legislación natural de la raza". Este punto se articula con la voluntad ya comentada de generar un registro civil autónomo, administrado por la propia Federación Araucana. Sin embargo, lo anterior no implica un desconocimiento frontal del rol legitimador del aparato estatal chileno. En la tabla de trabajos del Congreso se indica que "[legalmente] los Oficiales Civiles no podian negar de anotar el nombre de los padres de los nacidos en matrimonio por ritos indígenas" (AD11: 81). De esta forma vemos otro elemento estructural de la concepción política planteada por el Congreso: el recurso a la legalidad wingka como condición "externa" de legitimidad de la "legislación de la raza". Por último figura en el tercer punto la propuesta clasificatoria de Manquilef, por la cual se busca definir una nueva estructura de clases dentro de la sociedad mapuche que se determinaría en función del nivel de "civilización", es decir de educación, o más concretamente, de manejo de la escritura, de los sujetos mapuches. La clase así definida accedería a la igualdad plena de derechos comerciales con el resto de los ciudadanos chilenos. Esto equivale a negar el proyecto desarrollado por Aburto y su Federación Araucana de establecer un escritorio mapuche soberano, ya que desde la perspectiva de Manquilef, toda escritura mapuche pasaría automáticamente a inscribirse en un escritorio chileno único y total (proceso que equivale al de su propia biografía como escritor). Es en este mismo sentido que en el artículo del 11 de septiembre de 1926 publicado por el Diario Austral y defendiéndose de las críticas generadas por su proyecto de ley, declaraba que no era representante araucano: "solo represento al Partido Liberal Democrático de Cautín y a los indígenas civilizados de mi provincia" (El Diario Austral 11/9/1926). La clase de los indígenas civilizados comparte con el Partido Liberal Democrático el referir a un espacio exterior a toda forma de representatividad política mapuche, se trata de sujetos individuales actuando en el campo político general de la sociedad chilena. Sin embargo, vemos reaparecer aquel resto racial que finalmente continúa operando como argumento de legitimidad. Así, en el mismo artículo se justifica en última instancia haciendo referencia a lo más profundo de su corporalidad mapuche: "Mi sangre me dice que con este proyecto estoy sirviendo leal y efectivamente a la raza araucana" (Ibíd.). Cabe señalar que dos años antes, cuando recién se planteaba la necesidad de elaborar una nueva ley de radicación que salvaguardara los derechos mapuches sobre sus tierras (es decir las tierras asignadas por el Estado chileno a través de las leyes de 1866 y 1874), Manquilef, entonces presidente de la Sociedad Caupolicán y como miembro de la 
mesa directiva del IV Congreso Araucano protestaba "contra el gobierno por haber entregado la consulta de la ley sobre araucanos al Padre Capuchino Rvdo. Guido [Beck de Ramberga], habiendo entre los araucanos personas suficientemente capacitadas para informar sobre ella" ( $\mathrm{La} \mathrm{Na-}$ ción 26/12/1924). No resulta sorprendente que algunos meses más tarde fuera él mismo quien planteara una nueva "ley sobre los araucanos". Así vemos que al tiempo que plantea en su ley la disolución del cuerpo social y colectivo araucano en el continuo legal de la sociedad chilena, no trepida en recurrir a su propia condición de araucano para legitimarse como el legislador más pertinente para resolver los problemas de ese mismo cuerpo.

A la voz de la sangre en Manquilef corresponde en Aburto un concepto análogo y opuesto. El presidente de la Federación Araucana invocará a lo largo de su carrera, como fondo histórico y trascendente de legitimidad "las Virtudes de la raza" compuestas por la masa de caciques y de machis muertos. Estas se manifestarán bajo la forma manática de las "influencias espirituales", necesarias para la buena conducción de los asuntos políticos relacionados con el destino de su pueblo. Así cuando algunos años más tarde, en 1934, la Federación Araucana y el propio Aburto se encuentran sometidos a importantes presiones políticas derivadas de sus alianzas simultáneas con la Federación Obrera de Chile y con Arturo Alessandri (conflicto que concluirá con la relegación de Manuel Aburto a la isla de Chiloé en 1936), Aburto deberá desmarcarse de las declaraciones de Arturo Huenchullán, entonces diputado por el Partido Demócrata quien había dirigido duras críticas al gobierno tras la matanza de Ranquil. En esa ocasión, Alessandri había aprovechado su conflicto con Huenchullán para rechazar el diálogo con la totalidad del movimiento mapuche. Esta vez Huenchullán recurrirá a un gesto análogo al realizado por Manquilef en 1926, pero con el objetivo contrario de resguardar el movimiento mapuche de las furias del Presidente de la República, argumentando su calidad de representante del Partido Demócrata y no de la raza. Aburto aprovechará el gesto de Huenchullán para aconsejarle "que en lo sucesivo se acerque más a las virtudes de la raza para sus actuaciones políticas, antes que hacerlo por el Partido Demócrata porque se sabe que será un gran hombre y que necesita marchar de acuerdo con su raza para que no carezca de influencias espirituales" (Foerster \& Montecino 1988: 142). Así mientras para Manquilef lo mapuche actúa dentro, en un punto distante de su cuerpo, como marca genética o vestigio interior, en Aburto lo hace como un campo espiritual y colectivo que circunscribe y abarca toda la corporalidad mapuche.

\section{Conclusión: la raza como jurisprudencia}

Es en este espacio supraorgánico donde se deben buscar las bases de la moral y de la legislación natural de la raza invocadas por Aburto. Hemos visto cómo este espacio constituye finalmente un marcador de intra- 
ductibilidad por el cual se organiza todo el régimen de lealtades y de legitimidades puesto en escena por los Congresos Araucanos. Ahora bien, el otro gran referente levantado por Aburto y por el movimiento mapuche en general es el Estado, tanto bajo su aspecto coyuntural del gobierno de turno, como bajo su aspecto más trascendente de espacio legislador. De esta forma el debate desarrollado en el contexto del Congreso Araucano se estructura sobre la base de estos dos polos de heteronomía: las leyes de radicación dictadas por el Estado chileno en 1866 y 1874 y la legislación natural de la raza. De hecho, el recurso a un heterónomo como legitimador de las decisiones políticas parece ser una constante estructural en el desempeño político mapuche, o al menos en el marco de los Congresos Araucanos. Esto explica la gran importancia otorgada a los sueños por Manuel Aburto y a su registro sistemático en los archivos de la Federación Araucana. En este mismo sentido operan las influencias espirituales, bajo la forma de "noticias espirituales" que, por ejemplo, determinaban el apoyo de la Federación Araucana a uno u otro candidato a la Presidencia de la República. De esta forma la argumentación política reposaba sobre este heterónomo trascendente -influencias, noticias, o sueños-. Por su parte el Estado cumplía una función análoga asegurando la legitimidad de gestos y personajes dentro del espacio político mapuche. Recordemos que parte del carisma de Manuel Aburto residía justamente en la conexión que establece por su apellido con aquella institución de mediación estatal que fueron los Comisarios de Naciones. La lógica de los tratados participa seguramente de esta fuerza legitimadora que tiene la inscripción de lo heterogéneo en la coproducción del texto. Esto -y sin desestimar los evidentes cálculos estratégicos- puede explicar las reiteradas declaraciones de apoyo al gobierno hechas por Aburto a lo largo de su carrera, incluso siendo víctima de la represión por este mismo gobierno. En la entrevista que da en 1923 remonta genealógicamente esta relación de lealtad al Estado chileno:

“Mi abuelo [Luis Ayñamco] y su citado hijo, tío carnal mío según tradición y antecedentes que debe tener el Supremo Gobierno, por medio de la Intendencia de Valdivia, les cupo un gran papel en la pacificación de la Araucanía en esta provincia de Valdivia y jamás permitió que los indígenas faltasen el debido respeto al gobierno" (El Mercurio 20/1/1923).

En este marco las leyes de radicación dictadas en virtud de este mismo proceso de conquista, reviven algo del aura de los antiguos tratados.

Llegamos así al esquema anunciado de los Congresos Araucanos como empresas de escritura tendidas entre los polos heterogéneos de la escritura legal estatal y de la legislación natural de la raza en tanto legalidad intranscribible. Aburto, como Manquilef en su momento, junto con reconocer lo bien intencionado y lo positivo de las leyes de radicación, alegará por las profundas deficiencias en su aplicación. Es en este sentido que en 1919 Aburto criticaba el hecho que dichas leyes "ante el espíritu de la usurpación han resultado ser letra muerta" (Foerster \& Montecino, 1988: 33). En el otro extremo vibra la legislación natural de la raza como un orden de la vida pero desprovisto de letra. ¿Pero qué hace que entre la 
letra muerta y la vida iletrada prolifere semejante cantidad de escritura? Una respuesta posible se encuentra en la actividad judicial que constituye finalmente la labor principal de Manuel Aburto. Entre las actas y los acuerdos redactados con ocasión de los Congresos Araucanos, los archivos de la Federación Araucana abundan de casos y litigios en los que el propio Aburto opera como juez. Si los Congresos Araucanos funcionan como espacios intermitentes de discusión y resolución político-legislativa soberana, es en el ámbito judicial donde se logrará el mayor efecto práctico de soberanía. Aburto -heredando las cualidades del rol de Comisario de Naciones (Donoso \& Velasco, 1928: 39)- funciona tanto como mediador entre los particulares mapuches y las autoridades chilenas como entre los propios mapuches. Contamos con una serie de juicios llevados por Aburto, consecuente con la idea que los mapuches, o más precisamente los miembros de la Federación no debían recurrir a los tribunales chilenos, sino que a una entidad mapuche que tuviera en cuenta la legislación de la raza para dirimir sus conflictos. Así entre los casos más ilustrativos de esta función judicial autónoma se encuentra por ejemplo el "Reclamo $N^{\circ} 355$ de don Celestino Quileñan Colipi y de su señora doña Anjelina Meliñamco Reumai. -Sobre cuentos propios entre la raza Araucana, o más bien dicho, hechicerías", en el que el interesado declara:

En Loncoche, a treinta y uno de octubre de mil novecientos veintiocho [...] se le imputa el delito de calumnia que consiste en que ella es bruja, cuyo calificativo es bastante grave dentro de la legislación de la raza a la que pertenezco, y el que no puedo aceptar en forma alguna dentro del marco de la civilización, ya que mi citada mujer está muy lejos, entre nosotros los mapuches, de este concepto criminal ${ }^{10}$.

Fundándose en el referente no escrito de la legislación de la raza, Aburto va construyendo toda una jurisprudencia mapuche, a través de la cual se ejerce una forma de la soberanía, empresa que no pasó desapercibida para las autoridades que ordenaron el allanamiento de la sede de la Federación y la incautación de sus archivos ${ }^{11}$. El concepto de ley que está manejando Aburto al pensarla como una legislación de la raza funciona a primera vista como un oximorón: la legislación refiere al orden de lo construido, de una escritura, mientras que la raza que se le inscribe, al orden de lo dado. Sin embargo, en su inmanencia, esta ley funciona como una condición exterior a la constitución de la Raza, raza que se vuelve así el producto en proceso de una jurisprudencia en constante construcción. A través de este registro y de su archivo se produce un corpus que finalmente constituirá el referente último del cuerpo mapuche, hipostasiado en la noción de "Raza". En otras palabras, la Raza en Aburto es inseparable del registro

10. Archivo Siglo XX (ARNAD) Fondo Ministerio del Interior. Volumen 7321.

11. El oficial encargado de la operación escribía en su informe: "En cumplimiento de la Providencia $N^{\circ}$ 625, de 8-11-929, de esa Prefectura, me trasladé a Loncoche, en averiguaciones de las actividades que ejerce en la Provincia de Cautín, el indíjena Manuel Aburto Panguilef, por referencias y los documentos que acompaño, se desprende que sus actividades son amplias, y absolutas, entre los indíjenas, no conociéndo éstos más autoridad que al referido individuo, quién cobra honorarios por hacer algunas diligencias" (AD 1,1: 55). 
que la constituye como entidad histórica y política. A la Raza como resto nodular o vestigio indigesto en la corporalidad de un Manquilef disuelto en la universalidad de un humanismo ciudadano, le corresponde la Raza como entidad proteica, como escritura en acto, constituyéndose en las alianzas y los tratados que la reconocen.

\section{Fuentes de prensa}

El Mercurio 1923.- "Una entrevista con el presidente don Manuel Aburto Panguilef", en: El Mercurio, Santiago, 20 de enero de 1923.

El Diario Austral 1926.- "El proyecto de división de tierras indígenas se considera salvador para la raza. Entrevista con Manuel Manquilef"- en: El Diario Austral, Temuco, 11 de septiembre de 1926.

El Diario Austral 1926 “La ignorancia y mala fé de algunos elementos aborígenes oponen resistencia al proyecto de subdivision de la propiedad indígena. Entrevista a Manuel Manquilef", El Diario Austral, Temuco, 8 de septiembre 1926

El Diario Austral 1926.- "El proyecto de división de tierras indígenas se considera salvador para la raza. Entrevista a Manuel Manquilef"- en: El Diario Austral, Temuco, 11 de septiembre de 1926

El Diario Austral 1926. - "El proyecto gubernativo sobre radicación de indígenas ha dado origen a interesantes debates en el Parlamento. Entrevista a Manuel Manquilef"- en: El Diario Austral, Temuco, 25 de diciembre 1926. Orig. en: El Diario Ilustrado, Santiago.

La Nación 1924.- “Ayer se inauguró en la reducción de Huinquil cerca de Nva. Imperial, el Congreso Araucano"- en: La Nación, Santiago, 26 de diciembre de1924.

\section{Bibliografía}

Augusta, Félix José. 1996 (1916). Diccionario Araucano. Santiago: Cerro Manquehue.

Deleuze, Gilles. 1969. Logique du sens. París: Les Editions de Minuit.

Derrida, Jacques. 1967. De la grammatologie. París: Editions de Minuit.

Donoso, Ricardo \& Fanor Velasco. 1970 (1928). La propiedad austral. Santiago de Chile: ICIRA.

Foerster, Rolf \& Sonia Montecino 1988. Organizaciones, líderes y contiendas mapuches (1900-1970). Santiago de Chile: Centro de Estudios de la Mujer.

Guevara, Tomás \& Manuel Mañkelef. 2002 (1912). Kiñe mufü trokiñche ñi piel, historias de familias, siglo XIX.- Temuco \& Santiago: Colibris \& Liwen.

Hux, Meinrado 1991. Caciques huilliches y salineros. Buenos Aires: Marymar.

Laboratorio de Desclasificación Comparada. 2005. Anales de Desclasificación "La derrota del área cultural" 1 (LDC: Santiago).

Manquilef, Manuel. 1915. ¡Las tierras de Arauco! Temuco: Imprenta Modernista 
Menard, André \& Wilson Matuz. 2002. Entrevista a Germán Aburto Colihueque, Loncoche, diciembre 2001 y enero 2002, texto inédito.

Menard, André, 2004. "La escritura y su resto (el suplemento mapuche)". En: Revista de Historia Indígena 8 (Santiago: Departamento de Ciencias Históricas de la Universidad de Chile): 57-88.

Menard, André \& Jorge Pavez. 2005. “Los archivos del '29: derrotas y derroteros de la Federación Araucana". En: Anales de desclasificación 1 (1), 2005, Santiago: Laboratorio de Desclasificación Comparada.

Pavez, Jorge. 2003. "Mapuche ñi nütram chilkatun / Escribir la historia mapuche. Estudio posliminar de Kiñe mufü trokiñche ñi piel, historias de familias, siglo XIX". En Revista de Historia Indígena 7 (Santiago: Departamento de Ciencias Históricas de la Universidad de Chile): 7-53.

Virilio, Paul. 1977. Vitesse et politique. Essai de dromologie. París: Galilée. 